four-co-ordinated water lattice ${ }^{1}$. The proton magnetic resonance data support these ideas.

The dielectric method for studying water structure changes induced by macromolecules is more sensitive than the proton magnetic resonance technique. However, dielectric studies cannot be applied to solutions of high conductivity, whereas the proton magnetic resonance method is undisturbed by the presence of moderate amounts of electrolytes. This makes it possible to use the latter method for studies in vivo.

We wish to express our gratitude to Prof. Felix Bloch for helpful discussions, and to Dr. L. G. Allgen, Karolinska Institutet, Stockholm, for supplying one of the nucleic acid preparations.

\section{BERTIL JACOBSON*}

Weston A. Anderson

J AMES T. ARNold

Department of Physies,

Stanford University,

California. Jan. 30 .

* Fellow. Permanent address: Karolinska Institutet, Stockholm, Sweden.

'Jacobson, B., Nature, 172, 666 (1953).

${ }^{2}$ Gulland, J. M., Jordan, D. O., and Threlfall, C. J., J. Chem. Soc., 1129 (1947)

${ }^{3}$ Arnold, J. T., Dharmatti, S. S., and Packard, M. E., J. Chem. Phys., 19, 507 (1951).

\section{Synthesis of L-Amino-succinimide}

IN an attempt to account for the differences in the chemical and physiological properties of asparagine and glutamine, Steward and Thompson ${ }^{1}$ have suggested that the structure of asparagine is best described by a tautomeric system the major component of which is the monohydrate of amino-succinimide, while glutamine is best represented by the con. ventional straight-chain formulation. This theory has been questioned by Leach and Lindley², by Katz, Pasternak and Corey ${ }^{3}$, and by others. Steward and Thompson ${ }^{4}$ pointed out that the synthesis of amino-succinimide and a comparison of its properties in aqueous solution with a solution of asparagine would provide unequivocal evidence either for or against their hypothesis. We wish to report the synthesis of L-amino-succinimide.

In connexion with another investigation, we observed that the attempted saponification, under mild conditions, of carbobenzoxy-L-asparagine methyl ester $^{5}$ does not yield carbobenzoxy-L-asparagine. Instead the major product is a much weaker acid which we identified as carbobenzoxy-L-amino-succin. imide, m.p. $79-81^{\circ}, p K_{t}=9 \cdot 1$ in aqueous methanol, $[\alpha]_{D}^{24}-43^{\circ}$ (c. 1,95 per cent ethanol); (calc. for $\mathrm{C}_{12} \mathrm{H}_{12} \mathrm{~N}_{2} \mathrm{O}_{4}$ : C, $58.05 ; \mathrm{H}, 4.87 ; \mathrm{N}, 11.29$; amide- $\mathrm{N}$ : 5.65 ; found : $\mathrm{C}, 57.29 ; \mathrm{H}, 5 \cdot 17$; N, $11 \cdot 25$; amide-N, 5.50 per cent). Carbobenzoxy-Lamino-succinimide is also obtained from carbobenzoxy-L-isoasparagine methyl ester. Reaction of the imide with aqueous ammonia yields carbobenzoxyaspartic acid diamide. Prolonged treatment of the imide with alkali at room temperature, followed by hydrogenolysis to remove the carbobenzoxy group, results in the formation of a mixture of asparagine and isoasparagine.

Hydrogenolysis of carbobenzoxy-L-amino-succinimide yields L-amino-succinimide, m.p. approximately $144^{\circ}$ (decomp.), $[\alpha]_{D}^{24}-77^{\circ}$ (c. 1, methanol); (calc. for $\mathrm{C}_{4} \mathrm{H}_{6} \mathrm{~N}_{2} \mathrm{O}_{2}$ : C, $42 \cdot 10 ; \mathrm{H}, 5 \cdot 30 ; \mathrm{N}, 24 \cdot 6$; neut. equiv. 114 ; found : $\mathrm{C}, 42 \cdot 34 ; \mathrm{H}, 5 \cdot 28$; N, 24.85 per cent; neut. equiv. 118 (titrated with acid) ). Although L-amino-succinimide appears to be quite stable in the crystalline form, the compound is almost completely destroyed when a 1 per cent solution in phosphate buffer of $p H \mathbf{7 . 0}$ is heated for fourteen hours at $37^{\circ}$. Electrometric titration of an aqueous solution of $\mathrm{L}$-amino-succinimide shows the presence of a weakly acidic group with $p K_{a}=9 \cdot 0$, the imide-group, and a weakly basic group with $p K_{a}=5 \cdot 9$, the amino-group. A mixture of $\mathrm{L}$. asparagine and L-amino-succinimide can be separated readily by chromatography either with $n$-butanolacetic acid-water $(4: 1: 5)$ or with phenol saturated with water. It is thus clear that the properties of aqueous solutions of L-amino-succinimide and $\mathrm{L}$ asparagine are not similar.

Details of these investigations are being published elsewhere ${ }^{6}$.

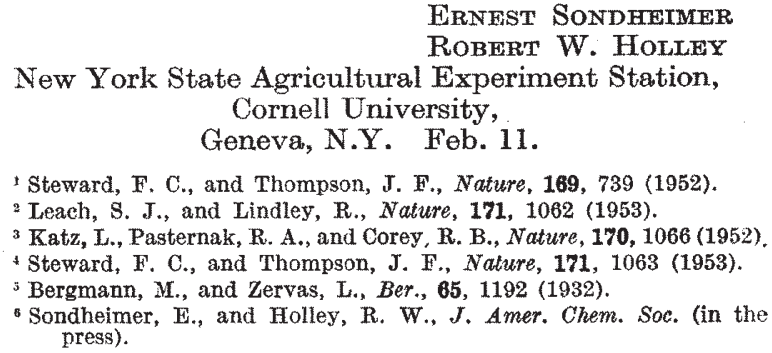

'Steward, F. C., and Thompson, J. F., Nature, 169, 739 (1952).

Leach, S. J., and Lindley, R., Nature, 171, 1062 (1953).

${ }^{3}$ Katz, L., Pasternak, R. A., and Corey, R. B., Nature, 170, 1066 (1952).

4 Steward, F. C., and Thompson, J. F., Nature, 171, 1063 (1953).

sergmann, M., and Zervas, L., Ber., 65, 1192 (1932).

Sondheimer, E., and Holley, R. W., J. Amer. Chem. Soc. (in the press).

IN our earlier consideration of the relationships of asparagine and glutamine, substances which are of great physiological importance, we directed attention to the interest which would be aroused by an unequivocal synthesis of amino-succinimide and whether "it proved to be, when hydrated, identical or different from asparagine" 1,2 .

Through the courtesy of Drs. Sondheimer and Holley, we have seen their paper ${ }^{3}$ and communication (above) on $\mathrm{t}$-amino-succinimide in advance of their publication. They are to be congratulated on what appears to be an elegant piece of synthetic work leading to derivatives of $\mathrm{L}$-amino-succinimide and to the pure substance, the properties of which are now described for the first time.

It is of great interest to us that the substance amino-succinimide: $(a)$ is stable in its crystalline anhydrous form; (b) can exist in aqueous solution and show by its titration curve the weakly acidic imide group and a weakly basic amino-group ; $(c)$ is distinguishable from $\mathrm{L}$-asparagine by its position on chromatograms, though, like that substance, it reacts brown with ninhydrin on paper ; $(d)$ is readily convertible, even by warming to $37^{\circ}$ at $p H ~ 7 \cdot 0$, to a mixture of asparagine and isoasparagine.

It is also of interest that in two ways, referred to by Sondheimer and Holley, there is a ready interconvertibility of the straight-chain form as it is supposed to occur in asparagine and the ring form as it occurs in amino-succinimide. This is shown by : (a) the hydrolysis of the methyl ester of carbobenzoxy asparagine yielding only carbobenzoxyamino-succinimide; (b) the combination of amino-succinimide with water to give asparagine and isoasparagine.

It is granted that the evidence of Sondheimer and Holley now is that asparagine and amino-succinimide can be distinguished. The important part of their evidence, in our view, is that which describes the properties of amino-succinimide in the free state and in solution. Apparently, amino-succinimide can exist unhydrated and distinguishable from asparagine, and when it com- 